\title{
FINITE FRATTINI FACTORS IN FINITELY GENERATED ABELIAN-BY-POLYCYCLIC GROUPS
}

\author{
JOHN C. LENNOX
}

\begin{abstract}
A criterion for a group to be finite is used to prove that subgroups of finitely generated Abelian-by-polycyclic groups are finite if their Frattini factor groups are finite.
\end{abstract}

In a previous paper [2] we considered finitely generated soluble groups and their subgroups in relation to the question whether they were finite if their Frattini factor groups were finite. Among other results we answered this question in the affirmative for subgroups of finitely generated Abelianby-nilpotent groups [2, Theorem B]. The class of all finitely generated Abelian-by-nilpotent groups is of course a proper subclass of the class of all finitely generated Abelian-by-polycyclic groups, since finitely generated nilpotent groups are polycyclic, but the converse does not hold. Now, although our methods in [2] depended on properties of finitely generated Abelian-by-polycyclic groups (more accurately, they depended on properties of Hall's class $\mathfrak{B}$ of Abelian groups where $B$ is in $\mathfrak{B}$ if and only if $B$ is an Abelian group which can be extended to a finitely generated group $G$ such that $G / B$ is polycyclic), they were not good enough to settle the question for the wider class of groups. Our object here is to close this gap by proving the

THEOREM. Subgroups of finitely generated Abelian-by-polycyclic groups are finite if their Frattini factor groups are finite.

The information we need here, supplementary to that contained in [2], is given in the following result which generalises Lemma 3 of [2] in a way which may be of interest in itself.

LEMMA. Let $K$ be a nilpotent normal subgroup of a group $H$. Then $H$ is finite if and only if $K$ is periodic, $H / \Phi(H) \cap K$ is finite and the centre of $K$ is reduced.

Received by the editors March 26, 1973.

AMS (MOS) subject classifications (1970). Primary 20E15, Secondary 20E20.

Key words and phrases. Frattini subgroup, finitely generated Abelian-by-polycyclic group.

(c) American Mathematical Society 1973 
Here $\Phi(H)$ denotes the Frattini subgroup of $H$. In [2] we were only able to establish this result under the additional hypothesis that $H / K$ was nilpotent.

ProOF OF THE LEMma. We shall adopt the notation of [2] throughout. Exactly as in the proof of Lemma 3 of [2] we can reduce to the case where $K$ is an elementary Abelian $p$-group for some prime $p$. Suppose $|H / \Phi(H)|=m$ and $|H| K \mid=n$. Consider a finite homomorphic image $\bar{H}$ of $H$. Let $d(\bar{H})$ be the number of generators of $\bar{H}$. Then $d(\bar{H}) \leqq m$ since $|\bar{H} / \Phi(\bar{H})| \leqq m$ and so by Schreier's theorem we have $d(R) \leqq 1+$ $(m-1) n$. Set $d=1+(m-1) n$. Then we have $|R| \leqq p^{d}$ and therefore $|\bar{H}| \leqq n p^{d}$. Thus we have proved that all finite images of $H$ are of bounded order. But $H$ is residually finite since it is a finite extension of the residually finite group $K$. It follows at once that $H$ is finite.

PROOF OF THE THEOREM. Suppose that $G$ is a finitely generated Abelian-by-polycyclic group and that $H$ is a subgroup of $G$ with $H / \Phi(H)$ finite. There exists an Abelian normal subgroup $A$ of $G$ such that $G / A$ is polycyclic. If $A=1$ then $G$ is polycyclic and therefore so also is $H$. By Theorem A of [2] it follows that $H$ is finite. Hence we may assume that $A \neq 1$. Furthermore, since $G$ satisfies the maximal condition for normal subgroups by [1, Theorem 5], we may assume that $H N / N$ is finite for all $1 \neq N \triangleleft G$. In particular, then, $H / H \cap A$ is finite. From Hall's theory of $\mathfrak{B}$-groups it follows exactly as in [2] that either $A$ is an elementary Abelian $p$-group for some prime $p$, in which case $H$ is finite by the Lemma or $A$ is torsion free, in which case we conclude that $H$ is finite by the argument which follows Lemma 5 of [2]. The theorem is proved.

We remark that it is not difficult to extend the theorem to the case where $G$ is a finite extension of a finitely generated Abelian-by-polycyclic group.

\section{REFERENCES}

1. P. Hall, Finiteness conditions for soluble groups, Proc. London Math. Soc. (3) 4 (1954), 419-436. MR 17, 344.

2. J. C. Lennox, Finite Frattini factors in finitely generated soluble groups, Proc. Amer. Math. Soc. 41 (1973), 356-360.

Department of Pure Mathematics, University College, Cardiff, Great BRITAIN 